\title{
ASYMPTOTIC BEHAVIOR OF POSITIVE SOLUTIONS OF SOME QUASILINEAR ELLIPTIC PROBLEMS
}

\author{
ZONGMING GUO AND LI MA
}

\begin{abstract}
We discuss the asymptotic behavior of positive solutions of the quasilinear elliptic problem $-\Delta_{p} u=a u^{p-1}-b(x) u^{q},\left.u\right|_{\partial \Omega}=0$ as $q \rightarrow p-1+0$ and as $q \rightarrow \infty$ via a scale argument. Here $\Delta_{p}$ is the $p$-Laplacian with $1<p<\infty$ and $q>p-1$. If $p=2$, such problems arise in population dynamics. Our main results generalize the results for $p=2$, but some technical difficulties arising from the nonlinear degenerate operator $-\Delta_{p}$ are successfully overcome. As a by-product, we can solve a free boundary problem for a nonlinear $p$-Laplacian equation.
\end{abstract}

\section{INTRODUCTION}

Let $\Omega \subset \mathbf{R}^{N}(N \geq 1)$ be a bounded smooth domain. We study the asymptotic behavior of positive solutions of the problem

$$
-\Delta_{p} u=a u^{p-1}-b(x) u^{q} \text { in } \Omega,\left.\quad u\right|_{\partial \Omega}=0
$$

for $q$ near $p-1$ and near $\infty$, respectively. Here $\Delta_{p} u=\operatorname{div}\left(|D u|^{p-2} D u\right)$ with $1<p<$ $\infty, b(x)$ is a nonnegative function in $C^{0}(\bar{\Omega}), a$ and $q$ are constants but $q$ is always greater than $p-1$.

Problem (1.1) with $p=2$ arises from mathematical biology and Riemannian geometry, and has attracted considerable interests; see, for example, [AT, AM, Da, DD, DDM, dP, FKLM, He, KW, Ma, Ou]. For general $p>1$, (1.1) has been considered in [CDG, DG1-2, Gu1-3, GZ, GZh, To]. The applications of (1.1) with $p>1$ can be found in [DG1]. We are concerned only with positive solutions of (1.1). We say $u$ a positive solution of (1.1) if $u \in W_{0}^{1, p}(\Omega) \cap C^{1}(\bar{\Omega})$ satisfies (1.1) in the weak sense with $u>0$ in $\Omega$.

When $b(x)$ is strictly positive on $\Omega$, it is known from [DG1] that for fixed $q>p-1$ it has no positive solution if $a \leq \lambda_{1}^{\Omega}$ and there is a unique positive solution $u=u_{a}$ when $a>\lambda_{1}^{\Omega}$, where $\lambda_{1}^{\Omega}$ denotes the first eigenvalue of the problem

$$
-\Delta_{p} u=\lambda|u|^{p-2} u,\left.\quad u\right|_{\partial \Omega}=0 .
$$

1991 Mathematics Subject Classification. Primary 35B45; Secondary 35J40.

Key words and phrases. Positive solutions, uniqueness, asymptotic behaviors. 
Moreover, $a \rightarrow u_{a}$ is continuous and strictly increasing as a function from $\left(\lambda_{1}^{\Omega}, \infty\right)$ to $C^{0}(\bar{\Omega})$ (with the natural order), and

$$
\lim _{a \rightarrow \lambda_{1}^{\Omega}+0} u_{a}(x)=0 \text { uniformly in } \bar{\Omega}
$$

$\lim _{a \rightarrow \infty} u_{a}(x)=\infty$ uniformly on any compact subset of $\Omega$.

When $b^{-1}(0):=\{x \in \Omega: b(x)=0\}$ is a proper subset of $\Omega$, the behavior of (1.1) is more complicated. Assume for simplicity that $b^{-1}(0)=\overline{\Omega_{0}} \subset \subset \Omega$, where $\Omega_{0}$ is open, connected and with smooth boundary. Then it is known from [DG1] that (1.1) has no positive solution unless $a \in\left(\lambda_{1}^{\Omega}, \lambda_{1}^{\Omega_{0}}\right)$, in which case there is a unique positive solution $u_{a}$ which varies continuously with $a$ and is strictly increasing in a. Moreover, $u_{a} \rightarrow 0$ uniformly on $\bar{\Omega}$ as $a \rightarrow \lambda_{1}^{\Omega}+0$, but as $a \rightarrow \lambda_{1}^{\Omega_{0}}, u_{a} \rightarrow \infty$ uniformly on $\overline{\Omega_{0}}$ and $u_{a} \rightarrow U$ uniformly on any compact subset $\bar{\Omega} \backslash \overline{\Omega_{0}}$, where $U$ is the unique minimal positive solution of the boundary blow-up problem

$$
-\Delta_{p} u=a u^{p-1}-b(x) u^{q}, \quad x \in \Omega \backslash \overline{\Omega_{0}} ;\left.\quad u\right|_{\partial \Omega}=0,\left.\quad u\right|_{\partial \Omega_{0}}=\infty .
$$

To understand the effect of the exponent $q$ on the unique positive solution of (1.1), we fix $p$ and $a$ and consider the cases that $q \rightarrow p-1+0$ and $q \rightarrow \infty$. In each case, we obtain a limiting problem which determines the asymptotical behavior of (1.1). The case when $p=2$ was studied by E.N. Dancer, Y. Du and L. Ma in [DDM].

We first recall some simple properties of the first eigenvalue of the $p$-Laplacian. Let $\phi \in L^{\infty}(\Omega)$ and denote by $\lambda_{1}^{\Omega}(\phi)$ the first eigenvalue of the problem

$$
-\Delta_{p} u+\phi|u|^{p-2} u=\lambda|u|^{p-2} u,\left.\quad u\right|_{\partial \Omega}=0 .
$$

Clearly, $\lambda_{1}^{\Omega}(0)=\lambda_{1}^{\Omega}$. It is known from Proposition 2.6 of [CDG] that $\lambda_{1}^{\Omega}\left(\phi_{n}\right) \rightarrow$ $\lambda_{1}^{\Omega}(\phi)$ whenever $\phi_{n} \rightarrow \phi$ in $L^{\infty}(\Omega)$, and when $\phi \leq \psi$ but $\phi \not \equiv \psi$ in $\Omega$, then $\lambda_{1}^{\Omega}(\phi)<$ $\lambda_{1}^{\Omega}(\psi)$. It follows from (ii) and (iii) of Proposition 2.6 of [CDG] that, when $b(x) \geq$ $\delta>0$ on $\Omega$, then $\lambda(\alpha):=\lambda_{1}^{\Omega}(\alpha b)$ is a strictly increasing function with $\lambda(0)=\lambda_{1}^{\Omega}$ and $\lambda(\alpha) \geq \lambda_{1}^{\Omega}(\alpha \delta):=\lambda_{1}^{\Omega}(0)+\alpha \delta \rightarrow \infty$ as $\alpha \rightarrow \infty$. Therefore, for any given $a>\lambda_{1}^{\Omega}$, there is a unique $\alpha>0$ such that

$$
a=\lambda_{1}^{\Omega}(\alpha b)
$$

We denote by $U_{\alpha}$ the corresponding positive normalized eigenfunction:

$$
-\Delta_{p} U_{\alpha}+\alpha b U_{\alpha}^{p-1}=a U_{\alpha}^{p-1}, \quad U_{\alpha}>0,\left.\quad U_{\alpha}\right|_{\partial \Omega}=0, \quad\left\|U_{\alpha}\right\|_{\infty}=1 .
$$

Here and in what follows, we use the notation $\|\cdot\|_{\infty}=\|\cdot\|_{L^{\infty}(\Omega)}$.

We can also consider the case that $b^{-1}(0)=\overline{\Omega_{0}}$ is not empty, we assume as before that $\Omega_{0} \subset \subset \Omega$ is open, connected and with smooth boundary. We will see from 
Proposition 4.1 below that $\lambda(\alpha)=\lambda_{1}^{\Omega}(\alpha b)$ is still strictly increasing and $\lambda(0)=\lambda_{1}^{\Omega}$, but

$$
\lim _{\alpha \rightarrow \infty} \lambda(\alpha)=\lambda_{1}^{\Omega_{0}}
$$

Thus for any given $a \in\left(\lambda_{1}^{\Omega}, \lambda_{1}^{\Omega_{0}}\right)$, there is a unique $\alpha>0$ satisfying (1.2) which determines a unique $U_{\alpha}$ through (1.3).

It is often important to determine what properties are retained when linear diffusion $(p=2)$ is replaced by nonlinear diffusion $(p \neq 2)$. In this paper we are concerned with this problem for (1.1), where the linear diffusion case, as mentioned above, has been studied extensively and is relatively well understood. We stress that it is not always possible to extend results from the case $p=2$ to the case $p \neq 2$ (for example, the existence and multiplicities of the eigenvalues of $-\Delta$ in $\Omega$ with Dirichlet boundary condition); and even if such extension is possible, one has to overcome many nontrivial technical difficulties arising from the nonlinear and degenerate operator $-\Delta_{p}$. Our main results of this paper are the following theorems.

Theorem 1.1. Suppose that $b(x)>0$ on $\bar{\Omega}$ and $a>\lambda_{1}^{\Omega}$. Let $u_{q}$ be the unique positive solution of (1.1). Then the following results hold:

(i) When $a<\lambda_{1}^{\Omega}(b)$, we have $u_{q} \rightarrow 0$ uniformly on $\bar{\Omega}$ as $q \rightarrow p-1+0$. Moreover, as $q \rightarrow p-1+0$,

$$
(q-p+1) \ln \left\|u_{q}\right\|_{\infty} \rightarrow \ln \alpha, \quad u_{q} /\left\|u_{q}\right\|_{\infty} \rightarrow U_{\alpha} \text { in } C^{1}(\bar{\Omega}),
$$

where $\alpha$ and $U_{\alpha}$ are determined by (1.2) and (1.3), respectively.

(ii) When $a>\lambda_{1}^{\Omega}(b)$, we have $u_{q} \rightarrow \infty$ uniformly on any compact subset of $\Omega$ as $q \rightarrow p-1+0$. Moreover, (1.4) holds.

(iii) When $a=\lambda_{1}^{\Omega}(b)$, we have $u_{q} \rightarrow c U_{1}$ in $C^{1}(\bar{\Omega})$ as $q \rightarrow p-1+0$, where $U_{1}$ is given by (1.3) with $\alpha=1$ and

$$
c=\exp \left(\int_{\Omega} b U_{1}^{p} \ln U_{1} d x / \int_{\Omega} b U_{1}^{q+1} d x\right) .
$$

For the case that $q \rightarrow \infty$, we have the following theorem.

Theorem 1.2. Suppose that $b(x)>0$ on $\bar{\Omega}$ and $a>\lambda_{1}^{\Omega}$. Let $u_{q}$ denote the unique positive solution of (1.1). Then $u_{q} \rightarrow v$ in $C^{1}(\bar{\Omega})$ as $q \rightarrow \infty$, where $v$ is the unique positive solution of

$$
-\Delta_{p} w=a \chi_{\{w<1\}} w^{p-1}, \quad w>0,\left.\quad w\right|_{\partial \Omega}=0,\|w\|_{\infty}=1 .
$$

The uniqueness of solutions of (1.5) is in the following proposition.

Proposition 1.3. For any $a \geq \lambda_{1}^{\Omega}$, (1.5) has a unique positive solution, and when $a<\lambda_{1}^{\Omega}$, (1.5) has no solution. 
When $\overline{\Omega_{0}}:=b^{-1}(0)$ is a nontrivial subset of $\Omega$, it turns out that the techniques in proving Theorems 1.1 and 1.2 are not enough. We need the following new ingredient for dealing with this case.

Lemma 1.4. Suppose that $\left\{u_{n}\right\} \subset C^{1}(\bar{\Omega})$ satisfies (in the weak sense) for some positive constant $\lambda$,

$$
-\Delta_{p} u_{n} \leq \lambda\left|u_{n}\right|^{p-2} u_{n}, \quad u_{n} \geq 0 \text { in } \Omega ;\left.\quad u_{n}\right|_{\partial \Omega}=0, \quad\left\|u_{n}\right\|_{\infty}=1 .
$$

Then it has a subsequence converging weakly in $W_{0}^{1, p}(\Omega)$ and strongly in $L^{m}(\Omega)$ for any $m \geq 1$, to some $u \in W_{0}^{1, p}(\Omega) \cap L^{\infty}(\Omega)$ with $u \neq \equiv 0$.

Theorem 1.5. Suppose that $\overline{\Omega_{0}}=b^{-1}(0)$ has nonempty interior which is connected with smooth boundary and $\Omega_{0} \subset \subset \Omega$. Let $a \in\left(\lambda_{1}^{\Omega}, \lambda_{1}^{\Omega_{0}}\right)$ and denote by $u_{q}$ the unique positive solution of (1.1). Then the conclusions (i)-(iii) in Theorem 1.1 hold.

When $b^{-1}(0) \neq \emptyset$ and $q \rightarrow \infty$, we have the following theorem.

Theorem 1.6. Suppose that $\overline{\Omega_{0}}=b^{-1}(0)$ has nonempty interior which is connected with smooth boundary and $\Omega_{0} \subset \subset \Omega$. Let $a \in\left(\lambda_{1}^{\Omega}, \lambda_{1}^{\Omega_{0}}\right)$ and denote by $u_{q}$ the unique positive solution of (1.1). Suppose that $q_{n} \rightarrow \infty$ and denote $u_{n}=u_{q_{n}}$. Then, subject to a subsequence, $u_{n} \rightarrow u$ in $L^{m}(\Omega)$ for all $m>1$, where $u \in K$ is a nontrivial nonnegative solution of the following variational inequality:

$$
\begin{gathered}
\int_{\Omega}|D u|^{p-2} D u \cdot D(v-u) d x-\int_{\Omega} a u^{p-1}(v-u) d x \geq 0, \quad \forall v \in K, \\
K:=\left\{w \in W_{0}^{1, p}(\Omega): w \leq 1 \text { a.e. in } \Omega \backslash \Omega_{0}\right\} .
\end{gathered}
$$

Theorem 1.5 concludes that, when $b^{-1}(0) \neq \emptyset$ and $q \rightarrow p-1$, the behavior of $u_{q}$ is the same as when $b^{-1}(0)=\emptyset$. But Theorem 1.6 concludes that this is not true for the case when $q \rightarrow \infty$. It is possible to show that for any given compact subset $D$ of $\Omega$, there exists a large $a_{D}$ such that the unique solution of (1.5) satisfies $w=1$ on $D$ when $a>a_{D}$. It is easily seen that for such $a$, and for those $\Omega_{0} \subset D$ satisfying $\lambda_{1}^{\Omega_{0}}>a$, if we let $u=w$ on $\bar{\Omega} \backslash \Omega_{0}$; and on $\Omega_{0}$, let $u$ equal the unique solution to $-\Delta_{p} u=a|u|^{p-2} u,\left.u\right|_{\partial \Omega_{0}}=1$, then $u$ solves (1.6).

\section{Proof of Theorem 1.1}

In this section we will give the proof of Theorem 1.1. The following lemma is well-known and easily obtained for $p=2$. Now, we present a proof for $p \neq 2$ by a scale argument.

Lemma 2.1. Let $\alpha$ be a constant and $w \in W_{0}^{1, p}(\Omega) \cap C^{1}(\bar{\Omega})$ be nonnegative with $w \not \equiv 0$, which satisfies, in the weak sense,

$$
-\Delta_{p} w=(a-\alpha b) w^{p-1},\left.\quad w\right|_{\partial \Omega}=0 .
$$


Then we necessarily have

$$
a=\lambda_{1}^{\Omega}(\alpha b) .
$$

Proof. We necessarily have $a \geq \lambda_{1}^{\Omega}(\alpha b)$ by the definition of $\lambda_{1}^{\Omega}(\alpha b)$ (see [CDG]). Moreover, by the equation of $w$, there exists $M>0$ such that

$$
-\Delta_{p} w+M w^{p-1} \geq 0 \text { in } \Omega \text {. }
$$

The strong maximum principle (see [Va]) then implies that $w>0$ in $\Omega$.

Now we show

$$
a=\lambda_{1}^{\Omega}(\alpha b) .
$$

Let $\phi_{1}^{\Omega}(\alpha b)$ with $\left\|\phi_{1}^{\Omega}(\alpha b)\right\|_{\infty}=1$ be the first eigenfunction corresponding to $\lambda_{1}^{\Omega}(\alpha b)$ and

$$
\beta=\sup \left\{\mu \in \mathbb{R}: w-\mu \phi_{1}^{\Omega}(\alpha b)>0 \text { in } \Omega\right\} .
$$

We have that

$$
w \geq \beta \phi_{1}^{\Omega}(\alpha b) \text { in } \Omega .
$$

(For simplicity, we denote $\phi_{1}^{\Omega}(\alpha b)$ by $\phi_{1}$ in the proof below.) We also know from [GW1] that $0<\beta<\infty$. Moreover,

$$
\begin{aligned}
& -\Delta_{p} w-\left\{-\Delta_{p}\left(\beta \phi_{1}\right)\right\}+\alpha b\left[w^{p-1}-\left(\beta \phi_{1}\right)^{p-1}\right] \\
& \geq \lambda_{1}^{\Omega}(\alpha b)\left[w^{p-1}-\left(\beta \phi_{1}\right)^{p-1}\right] \geq 0 .
\end{aligned}
$$

We will see that there exists $\delta_{1}>0$ such that $w \equiv \beta \phi_{1}$ in $\Omega_{\delta_{1}}$, where $\Omega_{\delta_{1}}=\{x \in \Omega$ : $\left.d(x, \partial \Omega)<\delta_{1}\right\}$. This clearly implies that

$$
\begin{aligned}
(a-\alpha b) w^{p-1} & =-\Delta_{p} w \\
& =-\Delta_{p}\left(\beta \phi_{1}\right)=\left[\lambda_{1}^{\Omega}(\alpha b)-\alpha b\right]\left(\beta \phi_{1}\right)^{p-1} \text { in } \Omega_{\delta_{1}}
\end{aligned}
$$

and thus

$$
a=\lambda_{1}^{\Omega}(\alpha b) .
$$

Now we show that there exists $\delta_{1}>0$ such that $w \equiv \beta \phi_{1}$ in $\Omega_{\delta_{1}}$. We first show that there exists $\eta \in \Omega$ where $w-\beta \phi_{1}$ vanishes. On the contrary, we have that $w>\beta \phi_{1}$ in $\Omega$. Since $a-\alpha b \in L^{\infty}(\Omega)$, by the strong maximum principle (see [Gu1, Va]), we have that $\frac{\partial w}{\partial n_{s}}<0$ and $\frac{\partial \phi_{1}}{\partial n_{s}}<0$ on $\partial \Omega$ (here $n_{s}$ is defined as in [GW1]). On the other hand, the compactness of $\partial \Omega$ implies that there exists $\delta_{1}>0$ and $\kappa>0$ such that

$$
\frac{\partial w}{\partial n_{s(x)}}<-\kappa \text { and } \frac{\partial \phi_{1}}{\partial n_{s(x)}}<-\kappa<0 \text { for } x \in \Omega_{\delta_{1}},
$$


where $n_{s(x)}$ is defined as in [GW1]. Therefore,

$$
t \frac{\partial w}{\partial n_{s(x)}}(x)+(1-t) \frac{\partial\left(\beta \phi_{1}\right)}{\partial n_{s(x)}}(x) \leq-\kappa \text { for } x \in \Omega_{\delta_{1}} \text { and all } t \in[0,1] .
$$

Hence, using the mean value theorem, we obtain

$$
\begin{aligned}
-\sum_{i, j} \frac{\partial}{\partial x_{i}}\left[a^{i j}(x) \frac{\partial\left(w-\beta \phi_{1}\right)}{\partial x_{j}}\right] & =-\Delta_{p} w-\left\{-\Delta_{p}\left(\beta \phi_{1}\right)\right\} \\
& =(a-\alpha b) w^{p-1}-\left[\lambda_{1}^{\Omega}(\alpha b)-\alpha b\right]\left(\beta \phi_{1}\right)^{p-1} \\
& \geq\left(\lambda_{1}^{\Omega}(\alpha b)-\alpha b\right)\left[w^{p-1}-\left(\beta \phi_{1}\right)^{p-1}\right] \geq 0 \text { in } \Omega_{\delta_{1}}
\end{aligned}
$$

where $a^{i j}(x)=\int_{0}^{1} \frac{\partial a^{i}}{\partial q_{j}}\left[t D w+(1-t) D\left(\beta \phi_{1}\right)\right] d t$ and $a^{i}=|q|^{p-2} q_{i}(i=1,2, \ldots N)$ for $q=\left(q_{1}, q_{2}, \ldots, q_{N}\right) \in \mathbb{R}^{N}$. Set

$$
-L \cdot=\sum_{i, j} \frac{\partial}{\partial x_{i}}\left[a^{i j}(x) \frac{\partial}{\partial x_{j}} \cdot\right] .
$$

Using (2.2), we see that $-L$ is a uniformly elliptic operator on $\Omega_{\delta_{1}}$. Consequently, we have

$$
\begin{gathered}
-L\left(w-\beta \phi_{1}\right) \geq 0 \text { in } \Omega_{\delta_{1}}, \\
w(x)>\beta \phi_{1}(x) \text { in } \Omega_{\delta_{1}}, \text { and } w-\beta \phi_{1}=0 \text { on } \partial \Omega\left(\text { part of } \partial \Omega_{\delta_{1}}\right) .
\end{gathered}
$$

By the Hopf's boundary point lemma of the uniformly elliptic operator, we obtain $\frac{\partial\left(w-\beta \phi_{1}\right)}{\partial n_{s}}<0$ on $\partial \Omega$. By arguments similar to those in [GW1], we see that there exists $\theta>0$ such that

$$
w(x) \geq(\beta+\theta) \phi_{1}(x) \text { for } x \in \Omega .
$$

This contradicts the definition of $\beta$.

To obtain our conclusion, we need to show that there exists $\tilde{\eta} \in \Omega_{\delta_{1}}$ where $w-\beta \phi_{1}$ vanishes. Otherwise, we can choose a domain $\Omega_{0} \subset \Omega$ with $\partial \Omega_{0} \subset \Omega_{\delta_{1}}$ and $\eta \in \Omega_{0}$ but $w-\beta \phi_{1} \geq \tau>0$ on $\partial \Omega_{0}$. Let $y=\beta \phi_{1}+\tau$. Then

$$
-\Delta_{p} w-\left\{-\Delta_{p} y\right\}=(a-\alpha b) w^{p-1}-\left(\lambda_{1}^{\Omega}\left(\alpha \phi_{1}\right)-\alpha b\right)\left(\beta \phi_{1}\right)^{p-1} \geq 0 \text { in } \Omega_{0}
$$

and

$$
w \geq y \text { on } \partial \Omega_{0} .
$$

The weak comparison principle (see [Gu1]) then implies that $w-\beta \phi_{1} \geq \tau>0$ in $\Omega_{0}$. But this contradicts the fact that $w-\beta \phi_{1}$ vanishes at $\eta \in \Omega_{0}$. This completes the proof.

Now we give the proof of Theorem 1.1. 
Set $M_{q}=\left\|u_{q}\right\|_{\infty}=\max _{\bar{\Omega}} u_{q}$. Then it is clear that the maximum is achieved in the interior of the domain $\Omega$, say at $x_{q} \in \Omega$. Using the equation for $u_{q}$ at the maximum point $x=x_{q}$ we claim

$$
a M_{q}^{p-1}-b\left(x_{q}\right) M_{q}^{q} \geq 0
$$

Hence,

$$
M_{q}^{q-p+1} \leq a / \min _{\bar{\Omega}} b .
$$

We need to explain a little here. Suppose $a M_{q}^{p-1}-b\left(x_{q}\right) M_{q}^{q}<0$. We can find a neighborhood $B_{\rho}\left(x_{q}\right)(\rho>0)$ such that $a u_{q}^{p-1}-b u_{q}^{q}<0$ in $B_{\rho}\left(x_{q}\right)$. Defining $w=M_{q}-u_{q}$, we see that $w \geq 0$ in $B_{\rho}\left(x_{q}\right)$ and $w$ attains its minimum at $x_{q}$. On the other hand,

$$
-\Delta_{p} w=\Delta_{p} u_{q}>0 \text { in } B_{\rho}\left(x_{q}\right) .
$$

This contradicts the strong maximum principle (see [Gu1]). Thus, our claim holds.

To understand the asymptotic behavior of $u_{q}$ as $q \rightarrow p-1+0$, we choose an arbitrary sequence $q_{n} \rightarrow p-1+0$ and use the notation

$$
u_{n}=u_{q_{n}}, \quad M_{n}=M_{q_{n}}, \quad \alpha_{n}=M_{q_{n}}^{q_{n}-p+1}, \quad w_{n}=u_{n} / M_{n} .
$$

Clearly $w_{n}$ satisfies the problem

$$
-\Delta_{p} w_{n}=a w_{n}^{p-1}-\alpha_{n} b w_{n}^{q_{n}},\left.\quad w_{n}\right|_{\partial \Omega}=0 .
$$

From (2.4) one sees that the right-hand side of (2.5) has a bound in $L^{\infty}(\Omega)$ which is independent of $n$. Thus, by the regularity of $-\Delta_{p}$ (see [Gu1]) we see that there is a subsequence of $\left\{w_{n}\right\}$ (still denoted by $\left\{w_{n}\right\}$ ) such that $w_{n} \rightarrow w$ in $C^{1}(\bar{\Omega})$. We may also assume that $\alpha_{n} \rightarrow \alpha$. Then from (2.5) we obtain, in the weak sense,

$$
-\Delta_{p} w=(a-\alpha b) w^{p-1},\left.\quad w\right|_{\partial \Omega}=0 .
$$

As $w$ is nonnegative with $\|w\|_{\infty}=1$, we see by Lemma 2.1 that $a=\lambda_{1}^{\Omega}(\alpha b)$ and hence $\alpha$ is uniquely determined by (1.2) and $w=U_{\alpha}$ given by (1.3). This implies that $\alpha_{n} \rightarrow \alpha$ and $w_{n} \rightarrow U_{\alpha}$ hold for the entire original sequences. Therefore, we have proved that $M_{q}^{q-p+1} \rightarrow \alpha$ and $u_{q} / M_{q} \rightarrow U_{\alpha}$ in $C^{1}(\bar{\Omega})$ as $q \rightarrow p-1+0$. This shows the validity of (1.4).

When $a<\lambda_{1}^{\Omega}(b)$, we must have $\alpha \in(0,1)$ and it follows from

$$
\lim _{q \rightarrow p-1+0}(q-p+1) \ln M_{q}=\ln \alpha
$$

that $M_{q} \rightarrow 0$ as $q \rightarrow p-1+0$. This proves Part (i) of Theorem 1.1.

When $a>\lambda_{1}^{\Omega}(b)$, we must have $\alpha>1$ and it follows from (2.6) that $M_{q} \rightarrow \infty$ as $q \rightarrow p-1+0$. To prove Part (ii) of Theorem 1.1, it remains to show that as 
$q \rightarrow p-1+0, u_{q}(x) \rightarrow \infty$ uniformly on any compact subset of $\Omega$. To this end, for any given large number $T$, we define $V=T U_{\alpha}$ and obtain

$$
\Delta_{p} V+a V^{p-1}-b V^{q}=b\left(\alpha V^{p-1}-V^{q}\right) .
$$

For those $x$ where $V(x) \leq 1, \alpha V^{p-1}-V^{q} \geq(\alpha-1) V^{p-1} \geq 0$; on the set $\{x \in \Omega$ : $V(x) \geq 1\}$, since $V^{q} \rightarrow V^{p-1}$ uniformly as $q \rightarrow p-1+0$, and since $\alpha V^{p-1}-V^{p-1} \geq$ $\alpha-1>0$, we can choose $\epsilon=\epsilon(T)>0$ small enough such that $\alpha V^{p-1}-V^{q}>0$ for all $q \in(p-1, p-1+\epsilon)$. Thus, for $q \in(p-1, p-1+\epsilon), V$ is a subsolution to (1.1). As any large positive constant is a supersolution to (1.1), its unique positive solution $u_{q}$ must satisfy $u_{q} \geq V=T U_{\alpha}$. This implies that as $q \rightarrow p-1+0, u_{q} \rightarrow \infty$ uniformly on any compact subset of $\Omega$ and Part (ii) of Theorem 1.1 is proved.

We consider now the case that $a=\lambda_{1}^{\Omega}(b)$. We have $\alpha=1$ and hence cannot derive a conclusion for $\lim _{q \rightarrow p-1+0} M_{q}$ from (2.6). Denote $w_{q}=u_{q} / M_{q}$. We see

$$
-\Delta_{p} w_{q}=a w_{q}^{p-1}-b M_{q}^{q-p+1} w_{q}^{q},\left.\quad w_{q}\right|_{\partial \Omega}=0 .
$$

Since $w_{q} \rightarrow U_{1}$ as $q \rightarrow p-1+0$ in $C^{1}(\bar{\Omega})$, and by the Hopf's boundary lemma, $\partial U_{1} / \partial \nu<0$ on $\partial \Omega$, we obtain $w_{q} / U_{1} \rightarrow 1$ uniformly on $\bar{\Omega}$. Thus,

$$
\begin{aligned}
\int_{\Omega}\left|D w_{q}\right|^{p-2} D w_{q} D U_{1} & =\int_{\Omega}\left|D U_{1}\right|^{p}+o(1) \\
& =\int_{\Omega}(a-b) U_{1}^{p}+o(1) \\
& =\int_{\Omega}(a-b) w_{q}^{p-1} U_{1}+o(1) .
\end{aligned}
$$

Thus, we obtain that as $q \rightarrow p-1+0$,

$$
\int_{\Omega}(a-b) w_{q}^{p-1} U_{1}=\int_{\Omega}\left(a w_{q}^{p-1}-b M_{q}^{q-p+1} w_{q}^{q}\right) U_{1} d x+o(1) .
$$

Hence

$$
\int_{\Omega} b\left(w_{q}^{p-1}-M_{q}^{q-p+1} w_{q}^{q}\right) U_{1} d x=o(1)
$$

and

$$
\int_{\Omega} \frac{M_{q}^{q-p+1}-1}{q-p+1} b w_{q}^{q} U_{1} d x=\int_{\Omega} \frac{1-w_{q}^{q-p+1}}{q-p+1} b w_{q}^{p-1} U_{1} d x+o(1) .
$$

We see that

$$
\left\|\ln w_{q}-\ln U_{1}\right\|_{\infty}=o(1)
$$

as $q \rightarrow p-1+0$. Therefore,

$$
\frac{1-w_{q}^{q-p+1}}{q-p+1} w_{q}^{p-1}=\frac{1-e^{(q-p+1)\left(\ln U_{1}+o(1)\right)}}{q-\underset{8}{p}+1} w_{q}^{p-1} \rightarrow U_{1}^{p-1} \ln U_{1}
$$


uniformly on $\bar{\Omega}$ as $q \rightarrow p-1+0$. From this, we see immediately that the right-hand side of (2.7) converges to

$$
\int_{\Omega} b U_{1}^{p} \ln U_{1} d x
$$

Thus,

$$
\lim _{q \rightarrow p-1+0} \int_{\Omega} \frac{M_{q}^{q-p+1}-1}{q-p+1} b w_{q}^{q} U_{1} d x=\int_{\Omega} b U_{1}^{p} \ln U_{1} d x
$$

and

$$
\lim _{q \rightarrow p-1+0} \frac{M_{q}^{q-p+1}-1}{q-p+1}=\int_{\Omega} b U_{1}^{p} \ln U_{1} d x / \int_{\Omega} b U_{1}^{q+1} d x .
$$

We show next that $c:=\lim _{q \rightarrow p-1+0} M_{q}$ exists and is uniquely determined by

$$
\ln c=\int_{\Omega} b U_{1}^{p} \ln U_{1} d x / \int_{\Omega} b U_{1}^{q+1} d x .
$$

We first claim that

$$
M_{*}:=\underline{\lim }_{q \rightarrow p-1+0} M_{q}>0, \quad M^{*}:=\varlimsup_{q \rightarrow p-1+0} M_{q}<\infty .
$$

Otherwise, we can find a sequence $\left\{q_{n}\right\}$ with $q_{n} \rightarrow p-1+0$ such that $M_{n}:=M_{q_{n}} \rightarrow 0$ or $M_{n} \rightarrow \infty$. in the former case, we deduce, for all large $n$,

$$
\frac{M_{n}^{q_{n}-p+1}-1}{q_{n}-p+1} \leq \frac{\epsilon^{q_{n}-p+1}-1}{q_{n}-p+1} \rightarrow \ln \epsilon
$$

as $n \rightarrow \infty$, for any given $\epsilon>0$. This leads to a contradiction to (2.8). In the latter case, we obtain, for all large $n$,

$$
\frac{M_{n}^{q_{n}-p+1}-1}{q_{n}-p+1} \geq \frac{M^{q_{n}-p+1}-1}{q_{n}-p+1} \rightarrow \ln M
$$

as $n \rightarrow \infty$, for any given $M>0$. This also leads to a contradiction to (2.8). Thus, $0<M_{*} \leq M^{*}<\infty$. For any given small $\epsilon>0$, a similar argument to the above leads to

$$
\begin{aligned}
& \ln \left(M_{*}+\epsilon\right) \geq \int_{\Omega} b U_{1}^{p} \ln U_{1} d x / \int_{\Omega} b U_{1}^{q+1} d x, \\
& \ln \left(M^{*}-\epsilon\right) \leq \int_{\Omega} b U_{1}^{p} \ln U_{1} d x / \int_{\Omega} b U_{1}^{q+1} d x .
\end{aligned}
$$

Thus we necessarily have

$$
M_{*}=M^{*}=c=\exp \left(\int_{\Omega} b U_{1}^{p} \ln U_{1} d x / \int_{\Omega} b U_{1}^{q+1} d x\right),
$$

and $u_{q} \rightarrow c U_{1}$ as $q \rightarrow p-1+0$ in $C^{1}(\bar{\Omega})$. This completes the proof of Theorem 1.1. 


\section{Proof of Theorem 1.2}

We still have (2.4). Let $\left\{q_{n}\right\}$ be a sequence with $q_{n} \rightarrow \infty$ as $n \rightarrow \infty$ and we use the notation in (2.5). We find that $w_{n}$ satisfies (2.5) whose right-hand side has a bound in $L^{\infty}(\Omega)$ which is independent of $n$. Thus, as in Section 2, subject to a subsequence, $w_{n} \rightarrow w$ in $C^{1}(\bar{\Omega})$.

The equation satisfied by $w_{n}$ can also be written as

$$
-\Delta_{p} w_{n}=a w_{n}^{p-1}-b u_{n}^{q_{n}-p+1} w_{n}^{p-1},\left.\quad w_{n}\right|_{\partial \Omega}=0 .
$$

From (2.4) we deduce

$$
0 \leq u_{n}^{q_{n}-p+1} \leq a / \min _{\bar{\Omega}} b
$$

Hence, by passing to a subsequence, we may assume that $b u_{n}^{q_{n}-p+1} \rightarrow \psi$ weakly in $L^{p^{\prime}}(\Omega)$ where $1 / p+1 / p^{\prime}=1$. Clearly we must have $0 \leq \psi \leq\|b\|_{\infty} a / \min _{\bar{\Omega}} b$. Passing to the weak limit in (3.1) we find that $w \in W_{0}^{1, p}(\Omega)$ is a nontrivial weak solution to the problem

$$
-\Delta_{p} w=(a-\psi) w^{p-1},\left.\quad w\right|_{\partial \Omega}=0, \quad\|w\|_{\infty}=1 .
$$

Since $a-\psi \in L^{\infty}(\Omega)$, we see from [Gu1] that $w \in C^{1}(\bar{\Omega})$. Moreover, there is $M>0$ such that

$$
-\Delta_{p} w+M w^{p-1} \geq 0 \text { in } \Omega .
$$

It follows from the strong maximum principle (see [Va]) that $w(x)>0$ for $x \in \Omega$.

From (2.4) we obtain

$$
M_{n} \leq\left(a / \min _{\bar{\Omega}} b\right)^{1 /\left(q_{n}-p+1\right)} \rightarrow 1 \text { as } n \rightarrow \infty
$$

It follows that $\varlimsup_{n \rightarrow \infty} M_{n} \leq 1$. If $\underline{\lim }_{n \rightarrow \infty} M_{n}<1$, then by passing to a subsequence, we may assume that $M_{n} \leq 1-\epsilon$ for all $n$ and some $\epsilon>0$. It follows then $u_{n}^{q_{n}-p+1} \leq$ $(1-\epsilon)^{q_{n}-p+1} \rightarrow 0$ as $n \rightarrow \infty$. Hence $\psi=0$ and $w$ is a positive solution to $-\Delta_{p} w=a w^{p-1},\left.w\right|_{\partial \Omega}=0,\|w\|_{\infty}=1$. This and Lemma 2.1 imply that $a=\lambda_{1}^{\Omega}$, contradicting our assumption that $a>\lambda_{1}^{\Omega}$. Thus we have proved that $M_{n} \rightarrow 1$ as $n \rightarrow \infty$. It also follows that $u_{n} \rightarrow w$ in $C^{1}(\bar{\Omega})$.

Let $\Omega_{1}:=\{x \in \Omega: w(x)<1\}$. Then for any $x \in \Omega_{1}$, we can find $\delta>0$ such that $u_{n}(x)<1-\delta$ for all large $n$. It follows that $0 \leq u_{n}(x)^{q_{n}-p+1} \leq(1-\delta)^{q_{n}-p+1} \rightarrow 0$ as $n \rightarrow \infty$. Thus we must have $\psi=0$ a.e. in $\Omega_{1}$. On the rest of $\Omega, w=1$ and we necessarily have $\Delta_{p} w=0$. (Here we regard $w$ as a member of $W_{0}^{1, p}(\Omega) \cap C^{1}(\bar{\Omega})$.) Thus from (3.3), we deduce $\psi=a$ a.e. on $\Omega \backslash \Omega_{1}$. Therefore, $w$ satisfies

$$
-\Delta_{p} w=a \chi_{\{w<1\}} w^{p-1},\left.\quad \begin{gathered}
w>0, \\
10
\end{gathered}\right|_{\partial \Omega}=0, \quad\|w\|_{\infty}=1 .
$$


This completes the proof.

\section{Proof of Proposition 1.3}

We first consider the case that $a<\lambda_{1}^{\Omega}$. Suppose (1.5) has a solution $w$ in this case. Then

$$
\int_{\Omega}|D w|^{p} d x \leq a \int_{\Omega} w^{p} d x<\lambda_{1}^{\Omega} \int_{\Omega} w^{p} d x .
$$

This contradicts the definition of $\lambda_{1}^{\Omega}$.

For $a=\lambda_{1}^{\Omega}$, we see that $\phi_{1}^{\Omega}$ is a solution of (1.5) with $\chi_{\left\{\phi_{1}^{\Omega}<1\right\}}=1$ a.e. in $\Omega$. Indeed, we have that

$$
a \int_{\Omega}\left(1-\chi_{\left\{\phi_{1}^{\Omega}<1\right\}}\right) w^{p-1} d x=0
$$

Since $w>0$ in $\Omega$, this gives us that $\chi_{\left\{\phi_{1}^{\Omega}<1\right\}}=1$ a.e. in $\Omega$.

For $a>\lambda_{1}^{\Omega}$, the proof of Theorem 1.2 implies that (1.5) has at least one solution.

In what follows, we only need to prove the uniqueness of solutions of (1.5). We do this by a scale argument similar to that in the proof of Lemma 2.1. What we do is to show that if $u_{1}$ and $u_{2}$ are two solutions of (1.5), then $u_{1} \geq u_{2}$ and $u_{2} \geq u_{1}$ in $\Omega$.

Let

$$
\begin{aligned}
& \beta=\sup \left\{\mu \in \mathbb{R}: u_{1}-\mu u_{2}>0 \text { in } \Omega\right\}, \\
& \gamma=\sup \left\{\mu \in \mathbb{R}: u_{2}-\mu u_{1}>0 \text { in } \Omega\right\} .
\end{aligned}
$$

Since $u_{1} \in C^{1}(\bar{\Omega}), u_{2} \in C^{1}(\bar{\Omega})$, we see from [GW1] that

$$
0<\beta<\infty, \quad 0<\gamma<\infty \text {. }
$$

Moreover, by the fact that $\left\|u_{i}\right\|_{\infty}=1(i=1,2)$, we see

$$
\beta \leq 1 \text { and } \gamma \leq 1
$$

The proof can be divided into two steps:

Step 1. The case that $0<\beta<1$ and $0<\gamma<1$.

Step 2. The case that $\beta=1$ or $\gamma=1$.

Note that if $\beta=1$ and $\gamma=1$, we see that $u_{1} \geq u_{2}$ and $u_{2} \geq u_{1}$ and hence $u_{2} \equiv u_{2}$ in $\Omega$. This is our conclusion.

Step 1. We know $0<\beta<1$ and $u_{1} \geq \beta u_{2}$ in $\Omega$. On the other hand, it follows from the Hopf's boundary lemma (see [Gu1, GW1]) that

For $\delta>0$ we let

$$
-\infty<\frac{\partial u_{1}}{\partial n_{s}}<0,-\infty<\frac{\partial u_{2}}{\partial n_{s}}<0 \text { on } \partial \Omega .
$$

$$
\Omega_{\delta}=\{x \in \Omega ; \operatorname{dist}(x, \partial \Omega) \leq \delta\}
$$


Since $\partial \Omega$ is compact, there are $\delta^{*}>0$ and $\kappa>0$ such that

$$
\frac{\partial u_{1}}{\partial n_{s(x)}}<-\kappa \text { and } \frac{\partial u_{2}}{\partial n_{s(x)}}<-\kappa \text { in } \Omega_{\delta^{*}} .
$$

We can choose $\delta^{*}>0$ small enough such that $u_{1}<1, u_{2}<1$ in $\Omega_{\delta^{*}}$. Thus $u_{1}$ and $u_{2}$ satisfy the problem

$$
-\Delta_{p} u_{i}=a u_{i}^{p-1} \text { in } \Omega_{\delta^{*}} \text { for } i=1,2 .
$$

We first show that there exists at least one point $x_{0} \in \Omega$ where $u_{1}-\beta u_{2}$ vanishes. On the contrary, we see $u_{1}>\beta u_{2}$ in $\Omega$. Therefore,

$$
-L\left(u_{1}-\beta u_{2}\right):=-\Delta_{p} u_{1}-\left\{-\Delta_{p}\left(\beta u_{2}\right)\right\}=a\left[u_{1}^{p-1}-\left(\beta u_{2}\right)^{p-1}\right] \text { in } \Omega_{\delta^{*}},
$$

where $-L$ is defined in the proof of Lemma 2.1 and thus is a uniformly elliptic operator in $\Omega_{\delta^{*}}$. It is easily seen from (3.5) that

$$
-L\left(u_{1}-\beta u_{2}\right)>0 \text { in } \Omega_{\delta^{*}}
$$

The Hopf's boundary lemma then implies that there exists $\theta>0$ such that

$$
u_{1}(x)-\beta u_{2}(x) \geq \theta \operatorname{dist}(x, \partial \Omega) \text { for } x \in \Omega_{\delta^{*}} .
$$

Since

$$
\ell_{1} \operatorname{dist}(x, \partial \Omega) \leq u_{2}(x) \leq \ell_{2} \operatorname{dist}(x, \partial \Omega),
$$

where $\ell_{2} \geq \ell_{1}>0($ see $[G W 1]),(3.6)$ implies

$$
u_{1}(x) \geq\left(\beta+\theta^{*}\right) u_{2}(x) \text { for } x \in \Omega_{\delta^{*}},
$$

where $\theta^{*}>0$. This and the fact that $u_{1}>\beta u_{2}$ in $\Omega$ imply

$$
u_{1} \geq\left(\beta+\theta^{* *}\right) u_{2} \text { in } \Omega .
$$

This contradicts the definition of $\beta$.

Now we claim that there exists a point in $\Omega_{\delta^{*}}$ where $u_{1}-\beta u_{2}$ vanishes. On the contrary, we can choose $\Omega_{0} \subset \subset \Omega$ with $\partial \Omega_{0} \subset \Omega_{\delta^{*}}$ and $\tau>0$ such that $u_{1}-\beta u_{2} \geq \tau$ on $\partial \Omega_{0}$. Moreover, there is at least one point in $\Omega_{0}$ where $u_{1}-\beta u_{2}$ vanishes. We can choose $\tau$ small enough so that $\beta+\tau<1$. Setting $w=\beta u_{2}+\tau$, we see from the fact $\left\|u_{i}\right\|_{\infty}=1(i=1,2)$ that

$$
\begin{aligned}
-\Delta_{p} u_{1}-\left\{-\Delta_{p} w\right\} & =a \chi_{\left\{u_{1}<1\right\}} u_{1}^{p-1}-a \chi_{\left\{u_{2}<1\right\}}\left(\beta u_{2}\right)^{p-1} \\
& \geq a \chi_{\left\{u_{1}<1\right\}} u_{1}^{p-1}-a \chi_{\left\{\beta u_{2}<1\right\}}\left(\beta u_{2}\right)^{p-1} \\
& =a \chi_{\left\{u_{1}<1\right\}} u_{1}^{p-1}-a\left(\beta u_{2}\right)^{p-1} \text { in } \Omega_{0} .
\end{aligned}
$$


Let $\mathcal{F}=\left\{x \in \Omega: u_{1}(x)=1\right\}$. We easily see that $\mathcal{F} \subset \subset \Omega_{0}$ and $u_{1} \geq w$ in $\mathcal{F}$. Thus, for $x \in \Omega_{0} \backslash \mathcal{F}$, we see that

$$
-\Delta_{p} u_{1}(x)-\left\{-\Delta_{p} w(x)\right\} \geq a\left[u_{1}^{p-1}(x)-\left(\beta u_{2}\right)^{p-1}(x)\right] \geq 0, \quad u_{1} \geq w \text { on } \partial \Omega_{0} \backslash \mathcal{F} \text {. }
$$

The weak comparison principle (see [Gu1]) implies that $u_{1} \geq w$ in $\overline{\Omega_{0} \backslash \mathcal{F}}$. This also implies $u_{1} \geq w\left(=\beta u_{2}+\tau\right)$ in $\Omega_{0}$, which contradicts the fact that there is at least one point in $\Omega_{0}$ where $u_{1}-\beta u_{2}$ vanishes. This contradiction implies that our claim holds. By the form of equation (3.5) and the strong maximum principle, we see

$$
u_{1} \equiv \beta u_{2} \text { in } \Omega_{\delta^{*}} .
$$

Since $0<\gamma<1$, the similar argument implies that

$$
u_{2} \equiv \gamma u_{1} \text { in } \Omega_{\delta^{*}}
$$

Therefore,

$$
u_{1} \equiv \beta \gamma u_{1} \text { in } \Omega_{\delta^{*}}
$$

and hence

$$
\beta \gamma=1
$$

But this contradicts the fact that $\beta \gamma<1$.

Step 2. We only consider the case that $\beta=1$. The case $\gamma=1$ and $\beta<1$ can be treated similarly. We see that $u_{1} \geq u_{2}$ in $\Omega$. On the other hand, we see $\chi_{\left\{u_{1}<1\right\}} \leq \chi_{\left\{u_{2}<1\right\}}$ in $\Omega$. Then

$$
-\Delta_{p} u_{1}-a \chi_{\left\{u_{2}<1\right\}} u_{1}^{p-1} \leq 0 \leq-\Delta_{p} u_{2}-a \chi_{\left\{u_{2}<1\right\}} u_{2}^{p-1} \text { on } \Omega .
$$

By a comparison principle (see Proposition 2.2 of [DG1]), wee see that

$$
u_{2} \geq u_{1} \text { in } \Omega \text {. }
$$

Therefore,

$$
u_{1} \equiv u_{2} \text { in } \Omega \text {. }
$$

This completes the proof of Proposition 1.3.

\section{Proof of Theorem 1.5}

We first see the fact mentioned in the introduction.

Proposition 4.1. Let $\left\{q_{k}\right\}$ be an increasing sequence of nonnegative functions in $C^{0}(\bar{\Omega})$ and $n \geq 1$ an integer number. Assume that $\Omega_{1}, \ldots, \Omega_{n}$ are smooth subdomains of $\Omega$ such that $\bar{\Omega}_{1}, \ldots, \bar{\Omega}_{n}$ are pair-wise disjoint and contained in $\Omega$. Moreover, suppose that

$$
q_{k} \equiv 0 \quad \text { on } \quad \cup_{i=1}^{n} \Omega_{i}
$$


and that

$$
\lim _{k \rightarrow \infty} \min _{x \in K} q_{k}(x)=\infty,
$$

for any compact subset $K$ of $\bar{\Omega} \backslash \cup_{i=1}^{n} \Omega_{i}$. Then,

$$
\lambda_{1}^{\Omega}\left(q_{k}\right) \uparrow \min _{1 \leq i \leq n} \lambda_{1}^{\Omega_{i}}
$$

as $k$ tends to infinity.

Proof. To keep the notation within reasonable bounds we only prove the case $n=2$. Without loss of generality we can assume that

$$
\lambda_{1}^{\Omega_{1}} \leq \lambda_{1}^{\Omega_{2}} .
$$

By the property that $\lambda_{1}^{\Omega}\left(q_{k}\right) \leq \lambda_{1}^{\Omega_{1}}\left(q_{k}\right)$ and (4.1), we see

$$
\lambda_{1}^{\Omega}\left(q_{k}\right) \leq \lambda_{1}^{\Omega_{1}} .
$$

Thus, $\lim _{k \rightarrow \infty} \lambda_{1}^{\Omega}\left(q_{k}\right)$ exists and lies below $\lambda_{1}^{\Omega_{1}}$. It suffices to show that for any $\epsilon>0$ there exists $k_{0} \geq 1$ such that

$$
0 \leq \lambda_{1}^{\Omega_{1}}-\lambda_{1}^{\Omega}\left(q_{k}\right)<\epsilon
$$

for all $k \geq k_{0}$. Fix $\epsilon>0$. By the continuous domain dependence and domain monotonicity of the Dirichlet principal eigenvalue for $i=1,2$ there exist smooth subdomains $\Omega_{i}^{\epsilon}$ containing $\Omega_{i}$ such that

$$
\begin{gathered}
\bar{\Omega}_{1}^{\epsilon} \cup \bar{\Omega}_{2}^{\epsilon} \subset \Omega, \quad \bar{\Omega}_{1}^{\epsilon} \cap \bar{\Omega}_{2}^{\epsilon}=\emptyset, \\
\lambda_{1}^{\Omega_{1}^{\epsilon}} \leq \lambda_{1}^{\Omega_{2}^{\epsilon}},
\end{gathered}
$$

and

$$
\lambda_{1}^{\Omega_{i}^{\epsilon}}<\lambda_{1}^{\Omega_{i}}<\lambda_{1}^{\Omega_{i}^{\epsilon}}+\epsilon,
$$

for $i=1,2$. Let $\varphi_{i}$ be the principal eigenfunction associated with $\lambda_{1}^{\Omega_{i}^{\epsilon}}$, which is unique up to positive multiplicative constants. By definition,

$$
-\Delta_{p} \varphi_{i}=\lambda_{1}^{\Omega_{i}^{\epsilon}} \varphi_{i}^{p-1} \text { in } \Omega_{i}^{\epsilon}, \quad \varphi_{i}=0 \text { on } \partial \Omega_{i}^{\epsilon},
$$

for $i=1,2$. We now choose two smooth subdomains, $\Omega_{1}^{*}$ and $\Omega_{2}^{*}$, such that

$$
\bar{\Omega}_{i} \subset \Omega_{i}^{*} \subset \bar{\Omega}_{i}^{*} \subset \Omega_{i}^{\epsilon}
$$

for $i=1,2$ and take any strictly positive function $\bar{u} \in C^{2}(\bar{\Omega})$ with $\Delta_{p} \bar{u} \in C^{0}(\bar{\Omega})$ satisfying

$$
\bar{u}=\varphi_{i} \text { in } \Omega_{i}^{*}
$$

for $i=1,2$, and

$$
\bar{u}>0 \text { on } \partial \Omega \text {. }
$$


As $\bar{u}>0$, it follows from (4.5) that

$$
-\Delta_{p} \bar{u}+q_{k}(x) \bar{u}^{p-1}+\left(\epsilon-\lambda_{1}^{\Omega_{1}}\right) \bar{u}^{p-1}>f_{k}(x) \text { in } \bar{\Omega}
$$

where

$$
f_{k}(x):=-\Delta_{p} \bar{u}+\left(q_{k}(x)-\lambda_{1}^{\Omega_{1}^{\epsilon}}\right) \bar{u}^{p-1}(x),
$$

for $x \in \bar{\Omega}$ and $k \geq 1$. Moreover, since $q_{k} \geq 0$ we find from (4.6) and (4.7) that

$$
f_{k} \geq 0 \text { in } \Omega_{1}^{*} \cup \Omega_{2}^{*}, \quad k \geq 1 .
$$

On the other hand, (4.2) implies that there exists $k_{0} \geq 1$ such that

$$
f_{k} \geq 0 \quad \text { in } \quad K:=\bar{\Omega} \backslash\left(\Omega_{1}^{*} \cup \Omega_{2}^{*}\right)
$$

for all $k \geq 1$. Thus, $f_{k} \geq 0$ in $\bar{\Omega}$ for any $k \geq k_{0}$ and hence

$$
-\Delta_{p} \bar{u}+q_{k}(x) \bar{u}^{p-1}+\left(\epsilon-\lambda_{1}^{\Omega_{1}}\right) \bar{u}^{p-1}>0 \text { in } \bar{\Omega}, \bar{u}>0 \text { on } \partial \Omega,
$$

for all $k \geq k_{0}$. Now we claim

$$
\lambda_{1}^{\Omega}\left(q_{k}+\epsilon-\lambda^{\Omega_{1}}\right)>0, \quad k \geq k_{0} .
$$

(4.10) implies

$$
\lambda_{1}^{\Omega_{1}}-\lambda_{1}^{\Omega}\left(q_{k}\right)<\epsilon, \quad k \geq k_{0}
$$

and the conclusion of this proposition holds.

Let $\sigma \leq 0$ be arbitrary. Then, for any $\alpha>0$ the function $\alpha \bar{u}$ is a super-solution of the problem

$$
-\Delta_{p} u=\left[\sigma-\left(q_{k}(x)+\epsilon-\lambda_{1}^{\Omega_{1}}\right)\right]|u|^{p-2} u+\omega \text { in } \Omega, \quad u=0 \text { on } \partial \Omega,
$$

where

$$
\omega=\alpha^{p-1} \min _{\bar{\Omega}}\left[-\Delta_{p} \bar{u}+\left(q_{k}(x)+\epsilon-\lambda_{1}^{\Omega_{1}}\right) \bar{u}^{p-1}\right]>0 .
$$

Suppose the problem

$$
-\Delta_{p} u+\left(q_{k}(x)+\epsilon-\lambda_{1}^{\Omega_{1}}\right)|u|^{p-2} u=\sigma|u|^{p-2} u \text { in } \Omega, \quad u=0 \text { on } \partial \Omega
$$

has a positive solution $u$. Then, there is a $M>\alpha$ such that

$$
u \leq M \bar{u} \text { in } \bar{\Omega} \text {. }
$$

It is clear that $\{\xi \bar{u}: \xi \in[\alpha, M]\}$ is a family of super-solution of (4.11) and $u$ is a sub-solution of (4.11). Thus, by a sweeping out result (see Remark 2.6 (2) of [GW2]), we see that

$$
u \leq \alpha \bar{u} \text { in } \bar{\Omega} .
$$


The arbitrariness of $\alpha$ implies that (4.12) does not admit a positive solution. Therefore,

$$
\lambda_{1}^{\Omega}\left(q_{k}+\epsilon-\lambda_{1}^{\Omega_{1}}\right)>0
$$

and our claim (4.10) holds. This completes the proof of this proposition.

To prove Theorem 1.5, we first present the proof of Lemma 1.4.

\section{Proof of Lemma 1.4}

It follows from the inequality satisfied by $u_{n}$ that

$$
\int_{\Omega}\left|D u_{n}\right|^{p} d x \leq \lambda \int_{\Omega} u_{n}^{p} d x \leq \lambda|\Omega| .
$$

This implies that $\left\{\left\|u_{n}\right\|_{W_{0}^{1, p}(\Omega)}\right\}$ is uniformly bounded. Thus it has a subsequence (still denoted by $\left\{u_{n}\right\}$ ) and $u \in W_{0}^{1, p}(\Omega)$ such that

$$
u_{n} \rightarrow u \text { weakly in } W_{0}^{1, p}(\Omega), \quad u_{n} \rightarrow u \text { strongly in } L^{p}(\Omega) \text {, as } n \rightarrow \infty \text {. }
$$

As $\left\|u_{n}\right\|_{\infty}=1, u_{n} \rightarrow u$ in $L^{p}(\Omega)$ implies $u_{n} \rightarrow u$ in $L^{m}(\Omega)$ for all $m \geq 1$. Clearly $0 \leq u \leq 1$.

It remains to show that $u \not \equiv 0$ in $\Omega$. Indeed, if $u \equiv 0$, then we have $u_{n} \rightarrow 0$ in $L^{m}(\Omega)$, for all $m \geq 1$. Let $v_{n}$ be the unique solution of the problem

$$
-\Delta_{p} v_{n}=\lambda u_{n}^{p-1} \text { in } \Omega, \quad v_{n}=0 \text { on } \partial \Omega .
$$

Then the regularity theory in [Gu1] implies that $v_{n} \rightarrow 0$ in $C^{1}(\bar{\Omega})$ as $n \rightarrow \infty$. On the other hand, it follows from the weak comparison principle that

$$
0 \leq u_{n} \leq v_{n} \text { in } \Omega
$$

Thus, $u_{n} \rightarrow 0$ in $L^{\infty}(\Omega)$, contradicting $\left\|u_{n}\right\|_{\infty}=1$. Therefore, we must have $u \not \equiv 0$. The proof is complete.

We are now in the position to give the proof of Theorem 1.5. We will mainly follow the lines of the proof of Theorem 1.1. The main difficulty is that the estimate (2.4) is of no use anymore and therefore it is unclear whether $\left\{\alpha_{n}\right\}$ is still bounded. We will use Lemma 1.4 to overcome this difficulty.

Let $q_{n}$ be an arbitrary sequence of numbers converging to $p-1+0$. We employ the notation in (2.5) and find that $w_{n}$ meets the conditions in Lemma 1.4. Hence, by passing to a subsequence, we may assume that $\left\|w_{n}\right\|_{W_{0}^{1, p}(\Omega)} \leq C, w_{n} \rightarrow w$ weakly in $W_{0}^{1, p}(\Omega)$, strongly in $L^{m}(\Omega)$ for any $m \geq 1$, and $w \neq \equiv 0$.

We claim that $\left\{\alpha_{n}\right\}$ is bounded. Otherwise, by passing to a subsequence, we may assume that $\alpha_{n} \rightarrow \infty$. Now we multiply (2.5), the equation satisfied by $w_{n}$, by $\phi / \alpha_{n}$ 
with $\phi \in C_{0}^{\infty}(\Omega)$ and integrate by parts. We obtain

$$
\left(\alpha_{n}\right)^{-1} \int_{\Omega}\left|D w_{n}\right|^{p-2} D w_{n} D \phi d x=\left(\alpha_{n}\right)^{-1} \int_{\Omega} a w_{n}^{p-1} \phi d x-\int_{\Omega} b w_{n}^{q_{n}} \phi d x .
$$

Notice that

$$
\begin{aligned}
\left.\left|\int_{\Omega}\right| D w_{n}\right|^{p-2} D w_{n} D \phi d x \mid & \leq\left(\int_{\Omega}\left|D w_{n}\right|^{p} d x\right)^{(p-1) / p}\left(\int_{\Omega}|D \phi|^{p}\right)^{1 / p} \\
& \leq C^{p-1}\left(\int_{\Omega}|D \phi|^{p} d x\right)^{1 / p} .
\end{aligned}
$$

Letting $n \rightarrow \infty$, we deduce

$$
\int_{\Omega} b w^{p-1} \phi d x=0 .
$$

As $\phi$ is arbitrary, this implies that $b w^{p-1}=0$ in $\Omega$. Hence, $w=0$ on $\Omega \backslash \Omega_{0}$. Since $w \in W_{0}^{1, p}(\Omega)$ and $\partial \Omega_{0}$ is smooth, this implies that $\left.w\right|_{\Omega_{0}} \in W_{0}^{1, p}\left(\Omega_{0}\right)$. Multiplying the equation for $w_{n}$ by an arbitrary $\phi \in C_{0}^{\infty}\left(\Omega_{0}\right)$ and integrating by parts, we obtain

$$
\int_{\Omega_{0}}\left|D w_{n}\right|^{p-2} D w_{n} \cdot D \phi d x=\int_{\Omega_{0}} a w_{n}^{p-1} \phi d x .
$$

Passing to $n \rightarrow \infty$ we obtain

$$
\int_{\Omega_{0}}|D w|^{p-2} D w \cdot D \phi d x=\int_{\Omega_{0}} a w^{p-1} \phi d x .
$$

Thus $\left.w\right|_{\Omega_{0}}$ is a weak solution of the problem

$$
-\Delta_{p} u=a u^{p-1},\left.\quad u\right|_{\partial \Omega_{0}}=0 .
$$

As $w=0$ on $\Omega \backslash \Omega_{0}$ and $w \not \equiv 0,\left.w\right|_{\Omega_{0}}$ is nonnegative and not identically zero. Hence we see by the scale argument as in the proof of Lemma 2.1 that $a=\lambda_{1}^{\Omega_{0}}$, contradicting our assumption that $a<\lambda_{1}^{\Omega_{0}}$. This proves our claim that $\left\{\alpha_{n}\right\}$ is bounded.

The rest of the proof follows from that of Theorem 1.1 except that to prove $u_{q} \geq T U_{\alpha}$, we use a comparison principle in [DG1] (which holds for $C^{1}$ functions).

\section{Proof of Theorem 1.6}

To prove this theorem, we use some fine properties of the limiting function $u$ in Lemma 1.4 and of functions in $W^{1, p}\left(\mathbb{R}^{N}\right)$ as mentioned in [DD] and [DDM]. Note that the fine properties of functions in $H^{1}\left(\mathbb{R}^{N}\right)$ given in [DD] and [DDM] hold for functions in $W^{1, p}\left(\mathbb{R}^{N}\right)$. This can be known from $[\mathrm{H}]$. We collect these fine properties in the following lemma.

Lemma 5.1. Let $u$ and $u_{n}$ be as in Lemma 1.4. Then the following conclusions hold: 
(i) $\tilde{u}(x)=\lim _{r \rightarrow 0} \int_{B_{r}(x)} u(y) d y /\left|B_{r}(x)\right|$ exists for each $x \in \Omega$, where $B_{r}(x)$ denotes the ball with center $x$ and radius $r$, and $\left|B_{r}(x)\right|$ stands for the volume of $B_{r}(x)$. Moreover, $u=\tilde{u}$ a.e. in $\Omega$.

(ii) $\tilde{u}$ is upper semi-continuous (u.s.c. for short) on $\Omega$, and for each $x_{0} \in \Omega$ and any given $\epsilon>0$, we can find a small ball $B_{r}\left(x_{0}\right) \subset \Omega$ such that for all large $n$,

$$
u_{n}(x) \leq \tilde{u}\left(x_{0}\right)+\epsilon, \quad \forall x \in B_{r}\left(x_{0}\right) .
$$

(iii) If $v \in W^{1, p}\left(\mathbb{R}^{N}\right)$, then $\tilde{v}(x)=\lim _{r \rightarrow 0} \int_{B_{r}(x)} v(y) d y /\left|B_{r}(x)\right|$ exists for all $x \in \mathbb{R}^{N}$ except possibly for a set of $(1, p)$-capacity 0 . Moreover, $\tilde{v}=v$ a.e. in $\mathbb{R}^{N}$ and if $\tilde{v}$ vanishes on a closed set $A$ in $\mathbb{R}^{N}$ (except for a subset of $A$ of capacity zero), then there exists a sequence of functions $\phi_{n} \in W^{1, p}\left(\mathbb{R}^{N}\right)$ such that each $\phi_{n}$ vanishes in a neighborhood of $A$ and $\phi_{n} \rightarrow \tilde{v}$ in $W^{1, p}\left(\mathbb{R}^{N}\right)$.

Now we give the proof of Theorem 1.6.

Let $q_{n}$ be a sequence converging to $\infty$ and use the notation in (2.5). Then as before, by Lemma 1.4 , subject to a subsequence, $w_{n} \rightarrow w$ weakly in $W_{0}^{1, p}(\Omega)$ and strongly in $L^{m}(\Omega)$ for any $m \geq 1$, and $w \not \equiv 0$.

Step 1. We show that $\left\{M_{n}\right\}$ is bounded.

Step 2. By passing to a subsequence, we may assume that $M_{n} \rightarrow c \in[0, \infty)$ as $n \rightarrow \infty$. We show that $c \geq 1$.

Step 3. We show that $w \leq 1 / c$ a.e. in $\Omega \backslash \Omega_{0}$.

Step 1. Since $a<\lambda_{1}^{\Omega_{0}}$, we can find a small $\delta$-neighborhood $\Omega_{\delta}$ of $\overline{\Omega_{0}}$ such that $a<\lambda_{1}^{\Omega_{\delta}}$. Let $\phi_{\delta}$ denote the normalized positive eigenfunction corresponding to $\lambda_{1}^{\Omega_{\delta}}$ :

$$
-\Delta_{p} \phi_{\delta}=\lambda_{1}^{\Omega_{\delta}} \phi_{\delta},\left.\quad \phi_{\delta}\right|_{\partial \Omega_{\delta}}=0, \quad\left\|\phi_{\delta}\right\|_{\infty}=1,
$$

and let $\psi \in C^{2}(\bar{\Omega})$ be an extension of $\left.\phi_{\delta}\right|_{\Omega_{\delta / 2}}$ to $\bar{\Omega}$ such that $\Delta_{p} \psi \in C(\bar{\Omega})$ and $\eta:=\min _{\bar{\Omega}} \psi>0$. We find, for any positive constant $T$,

$$
\begin{gathered}
\Delta_{p}(T \psi)+a(T \psi)^{p-1}-b(T \psi)^{q} \leq\left(a-\lambda_{1}^{\Omega_{\delta}}\right)(T \psi)^{p-1}<0, \quad \text { in } \Omega_{\delta / 2}, \\
\Delta_{p}(T \psi)+a(T \psi)^{p-1}-b(T \psi)^{q}=T^{p-1}\left(\Delta_{p} \psi+a \psi^{p-1}\right)-b T^{q} \psi^{q}, \quad \text { in } \Omega \backslash \Omega_{\delta / 2} .
\end{gathered}
$$

Let $\xi=\inf _{\Omega \backslash \Omega_{\delta / 2}} b$ and

$$
T_{q}:=\left[\xi^{-1} \sup _{\Omega}\left(\Delta_{p} \psi+a \psi^{p-1}\right) \eta^{-q}\right]^{1 /(q-p+1)} .
$$

We easily see that for $T=T_{q}$,

$$
\Delta_{p}(T \psi)+a(T \psi)^{p-1}-b(T \psi)^{q} \leq 0, \text { in } \Omega .
$$

Therefore, $Q_{q} \psi$ is a supersolution of (1.1). As (1.1) has arbitrarily small positive subsolutions, its unique positive solution $u_{q}$ must satisfy $u_{q} \leq T_{q} \psi$. Clearly $T_{q} \rightarrow 1 / \eta$ 
as $q \rightarrow \infty$. Thus, for any $q_{0}>p-1,\left\{M_{q}: q \geq q_{0}\right\}$ is bounded. In particular, $\left\{M_{n}\right\}$ is bounded. This completes the proof of Step 1.

Step 2. Let $v_{n}$ be the unique solution of

$$
-\Delta_{p} v=a v^{p-1}-\|b\|_{\infty} v^{q_{n}},\left.\quad v\right|_{\partial \Omega}=0 .
$$

By Theorem 1.2 we know $\left\|v_{n}\right\|_{\infty} \rightarrow 1$. On the other hand, a comparison argument (see [DG1]) shows $u_{n} \geq v_{n}$. Hence $c \geq 1$.

Step 3. $w \leq 1 / c$ a.e. in $\Omega \backslash \Omega_{0}$. Otherwise the set $\left\{x \in \Omega \backslash \Omega_{0}: w(x)>1 / c\right\}$ has positive measure and we can find some $c_{1}>1 / c$ such that $\Omega_{1}:=\left\{x \in \Omega \backslash \Omega_{0}\right.$ : $\left.w(x) \geq c_{1}\right\}$ has positive measure. As $w_{n} \rightarrow w$ in $L^{p}(\Omega)$, by passing to a subsequence, $w_{n} \rightarrow w$ a.e. in $\Omega$. Hence, by Egorov's theorem, we can find a subset of $\Omega_{1}$, say $\Omega_{2}$ which has positive measure and such that $w_{n} \rightarrow w$ uniformly on $\Omega_{2}$. It follows that $u_{n} \rightarrow c w$ uniformly on $\Omega_{2}$. Thus, there exists $\epsilon>0$ such that for all large $n$, $u_{n} \geq 1+\epsilon$ on $\Omega_{2}$.

Let $\phi \in C_{0}^{\infty}(\Omega)$ be an arbitrary nonnegative function, and multiplying the equation for $w_{n}$ by $\phi$ and integrating over $\Omega$, we obtain

$$
\int_{\Omega}\left|D w_{n}\right|^{p-2} D w_{n} \cdot D \phi d x=a \int_{\Omega} w_{n}^{p-1} \phi d x-\int_{\Omega} b u_{n}^{q_{n}-p+1} w_{n}^{p-1} \phi d x .
$$

Hence, for all large $n$,

$$
\begin{aligned}
(1+\epsilon)^{q_{n}-p+1} \int_{\Omega_{2}} b w_{n}^{p-1} \phi d x & \leq \int_{\Omega_{2}} b u_{n}^{q_{n}-p+1} w_{n}^{p-1} \phi d x \\
& \leq-\int_{\Omega}\left|D w_{n}\right|^{p-2} D w_{n} \cdot D \phi d x+a \int_{\Omega} w_{n}^{p-1} \phi d x .
\end{aligned}
$$

Dividing the above inequality by $(1+\epsilon)^{q_{n}-p+1}$ and letting $n \rightarrow \infty$, we deduce

$$
\int_{\Omega_{2}} b w^{p-1} \phi d x=0
$$

It follows that $w=0$ a.e. in $\Omega_{2}$, contradicting the assumption that $w \geq c_{1}$ there. This proves Step 3.

Using $u_{n}=M_{n} w_{n}$ and denoting $\hat{u}=c w$, we see from Lemma 5.1 and Steps 1-3 above that the following result holds:

Lemma 5.2. (i) $\left\{\left\|u_{n}\right\|_{\infty}\right\}$ is bounded.

(ii) Subject to a subsequence, $u_{n} \rightarrow \hat{u}$ weakly in $W_{0}^{1, p}(\Omega)$ and strongly in $L^{m}(\Omega)$, $\forall m \geq 1$.

(iii) $\hat{u} \leq 1$ a.e. in $\Omega \backslash \Omega_{0}$ and $\|\hat{u}\|_{\infty} \geq 1$.

(iv) $\tilde{u}(x):=\lim _{r \rightarrow 0} \int_{B_{r}(x)} \hat{u}(y) d y /\left|B_{r}(x)\right|$ exists for every $x \in \Omega$.

(v) $\tilde{u}(x)$ is u.s.c. on $\Omega$ and $\tilde{u}=\hat{u}$ a.e. in $\Omega$. 
(vi) For each $x_{0} \in \Omega$ and any given $\epsilon>0$, we can find a small ball $B_{r}\left(x_{0}\right) \subset \Omega$ such that for all large $n$,

$$
u_{n}(x) \leq \tilde{u}\left(x_{0}\right)+\epsilon, \quad \forall x \in B_{r}\left(x_{0}\right) .
$$

We are now ready to complete the proof of Theorem 1.6. The main idea is similar to that in the proof of Theorem 1.6 of [DDM]. Multiplying the equation for $u_{n}$ by $\phi \in C_{0}^{\infty}(\Omega)$, we deduce

$$
\int_{\Omega}\left|D u_{n}\right|^{p-2} D u_{n} \cdot D \phi d x=a \int_{\Omega} u_{n}^{p-1} \phi d x-\int_{\Omega} b(x) u_{n}^{q_{n}} \phi d x .
$$

It follows that, subject to a subsequence,

$$
\lim _{n \rightarrow \infty} \int_{\Omega} b(x) u_{n}^{q_{n}} \phi d x=-\int_{\Omega}|D \hat{u}|^{p-2} D \hat{u} \cdot D \phi d x+a \int_{\Omega} \hat{u}^{p-1} \phi d x, \quad \forall \phi \in C_{0}^{\infty}(\Omega) .
$$

Clearly the right-hand side of (5.1) defines a continuous linear functional on $W_{0}^{1, p}(\Omega)$ :

$$
\mathcal{F}(\phi)=-\int_{\Omega}|D \hat{u}|^{p-2} D \hat{u} \cdot D \phi d x+a \int_{\Omega} \hat{u}^{p-1} \phi d x .
$$

Arguments similar to those in the proof of Theorem 1.6 of [DDM] imply

$$
\begin{gathered}
\mathcal{F}(\phi) \geq 0, \forall \phi \in W_{0}^{1, p}(\Omega) \text { satisfying } \phi \geq 0 \text { a.e. on } \Omega \backslash \Omega_{0}, \\
\mathcal{F}(\phi)=0, \forall \phi \in W_{0}^{1, p}(\Omega) \text { satisfying } \operatorname{supp}(\phi) \subset \Omega_{0} \cup\{\hat{u}<1\} .
\end{gathered}
$$

The rest of the proof is same as that of Theorem 1.6 of [DDM], we present it for completeness. By Lemma 5.2 (iii), we easily see that $\tilde{u} \leq 1$ on the open set $\Omega \backslash \overline{\Omega_{0}}$. We show next that $\tilde{u}$ is close to 0 near $\partial \Omega$ and $\tilde{u} \leq 1$ on $\partial \Omega_{0}$. By Lemma 5.2 (i), we can find $M>0$ such that $a u_{n}^{p-1}<M$ on $\Omega$ for all $n \geq 1$. Therefore

$$
-\Delta_{p} u_{n}=a u_{n}^{p-1}-b(x) u_{n}^{q_{n}} \leq M \text { on } \Omega .
$$

If $V$ is given by

$$
-\Delta_{p} V=M \text { in } \Omega,\left.\quad V\right|_{\partial \Omega}=0,
$$

we obtain by the comparison principle in (see [Gu1]) that $u_{n} \leq V$. It follows that $\tilde{u} \leq V$. Therefore, $\tilde{u}$ is close to 0 near $\partial \Omega$. Since $\tilde{u} \leq 1$ on $\Omega \backslash \overline{\Omega_{0}}$, we must have $\tilde{u} \leq 1$ on $\partial \Omega_{0}$ except possibly for a set of capacity zero (see, [DDM]).

From the above analysis, we see that it is possible to choose $\phi \in C_{0}^{\infty}(\Omega)$ such that $0 \leq \phi \leq 1$ on $\Omega$ and $\phi=1$ on a $\delta$-neighborhood $N_{\delta}$ of $\{\hat{u}=1\}$. Let $v \in K$ be arbitrary and denote $\hat{v}=\max \{v, \phi\}$. Clearly $0 \leq \hat{v}-v \in W_{0}^{1, p}(\Omega)$. Thus, by (5.2),

$$
\begin{gathered}
\int_{\Omega}|D \hat{u}|^{p-2} D \hat{u} \cdot D(v-\hat{u}) d x-a \int_{\Omega} \hat{u}^{p-1}(v-\hat{u}) d x=-\mathcal{F}(v-\hat{u}) \\
=\mathcal{F}(\hat{v}-v)+\mathcal{F}(\hat{u}-\hat{v}) \geq \underset{20}{\geq \mathcal{F}(\hat{u}-\hat{v}) .}
\end{gathered}
$$


Denote $u^{*}=\hat{u}-\hat{v}$. Clearly $u^{*} \in W_{0}^{1, p}(\Omega)$. Now we choose $\psi \in C_{0}^{\infty}(\Omega)$ satisfying $0 \leq \psi \leq 1$ on $\Omega, \psi=0$ on $\Omega \backslash N_{(2 / 3) \delta}, \psi=1$ on $N_{(1 / 3) \delta}$. Then clearly

$$
\operatorname{supp}\left((1-\psi) u^{*}\right) \subset \bar{\Omega} \backslash N_{(1 / 3) \delta} \subset\{\tilde{u}<1\} \cup \Omega_{0} .
$$

Hence, by (5.3),

$$
\mathcal{F}\left(u^{*}\right)=\mathcal{F}\left((1-\psi) u^{*}\right)+\mathcal{F}\left(\psi u^{*}\right)=\mathcal{F}\left(\psi u^{*}\right) .
$$

As $\psi=0$ on $\Omega \backslash N_{(2 / 3) \delta}$, and $\hat{v}=\max \{v, \phi\}=1$ a.e. on $N_{\delta}$, we find that $\psi u^{*}=$ $\psi(\tilde{u}-1)$ a.e. on $\Omega$. Since $\psi(\hat{u}-1)$ is zero outside $N_{(2 / 3) \delta}$ it can be regarded as a member of $W^{1, p}\left(\mathbb{R}^{N}\right)$. It is easily seen that the representative of $\psi(\hat{u}-1)$ obtained through the limiting process in Lemma 5.1 (iii) is $\psi(\tilde{u}-1)$. Thus we obtain

$$
\mathcal{F}\left(u^{*}\right)=\mathcal{F}\left(\psi u^{*}\right)=\mathcal{F}(\psi(\tilde{u}-1)) .
$$

As $\tilde{u} \leq 1$ on $\bar{\Omega} \backslash \Omega_{0}$ and is u.s.c., we find that the set $A_{1}:=\{\tilde{u}=1\} \cap\left(\bar{\Omega} \backslash \Omega_{0}\right)$ is closed. Let $A_{2}:=\mathbb{R}^{N} \backslash N_{(2 / 3) \delta}$ and $A=A_{1} \cup A_{2}$. We know that $\psi(\tilde{u}-1)$ vanishes on the closed set $A$ (except possibly for a set of capacity zero) and so by Lemma 5.1 (iii), it can be approximated in the $W^{1, p}\left(\mathbb{R}^{N}\right)$ norm by $\phi_{n} \in W^{1, p}\left(\mathbb{R}^{N}\right)$ with each $\phi_{n}$ vanishing in a neighborhood of $A$. Therefore, $\operatorname{supp}\left(\phi_{n}\right) \subset\{\tilde{u}<1\} \cup \Omega_{0}$, and by (5.3), $\mathcal{F}\left(\phi_{n}\right)=0$. It follows that

$$
\mathcal{F}\left(u^{*}\right)=\mathcal{F}(\psi(\tilde{u}-1))=\lim _{n \rightarrow \infty} \mathcal{F}\left(\phi_{n}\right)=0 .
$$

We thus obtain

$$
\int_{\Omega}|D \hat{u}|^{p-2} D \hat{u} \cdot D(v-\hat{u}) d x-a \int_{\Omega} \hat{u}^{p-1}(v-\hat{u}) d x \geq 0, \forall v \in K .
$$

That is to say that $\hat{u} \in K$ is a solution of (1.6). This finishes our proof of Theorem 1.6 .

\section{Comments}

Just as in $[\mathrm{DDM}]$, we believe that the following result is true. However, we can only prove it in the special case when $N \leq 2$.

Conjecture 6.1: Suppose that $\left\{u_{n}\right\} \subset C^{1}(\bar{\Omega})$ satisfies (in the weak sense) for some positive constant $\lambda$,

$$
-\Delta_{p} u_{n} \leq \lambda\left|u_{n}\right|^{p-2} u_{n}, \quad u_{n} \geq 0 \text { in } \Omega ;\left.\quad u_{n}\right|_{\partial \Omega}=0, \quad\left\|u_{n}\right\|_{\infty}=1 .
$$

Then it has a subsequence converging weakly in $W_{0}^{1, p}(\Omega)$ and strongly in $L^{m}(\Omega)$ for any $m>1$, to some $u$ with $\|u\|_{\infty}=1$.

Proof of Conjecture 6.1 when $N \leq 2$ : 
It follows from the inequality satisfied by $u_{n}$ that

$$
\int_{\Omega}\left|D u_{n}\right|^{p} d x \leq \lambda \int_{\Omega} u_{n}^{p} d x \leq \lambda|\Omega|
$$

This implies that $\left\{\left\|u_{n}\right\|_{W_{0}^{1, p}(\Omega)}\right\}$ is uniformly bounded. Thus it has a subsequence (still denoted by $\left\{u_{n}\right\}$ ) and $u \in W_{0}^{1, p}(\Omega)$ such that

$$
u_{n} \rightarrow u \text { weakly in } W_{0}^{1, p}(\Omega), \quad u_{n} \rightarrow u \text { strongly in } L^{p}(\Omega) \text {, as } n \rightarrow \infty \text {. }
$$

Note that once we a uniform $L^{\infty}$ bound on $u_{n}$, we have $u_{n} \rightarrow u$ strongly in $L^{m}(\Omega)$ for any $m>1$.

To show that $\|u\|_{\infty}=1$, we argue by contradiction. Assume that $\|u\|_{\infty}=1-\epsilon<$ 1. We will show that $\left\|u_{n}\right\|_{\infty}<1$ for large $n$, which gives us a contradiction.

Solve

$$
-\Delta_{p} v_{n}=\lambda\left|u_{n}\right|^{p-2} u_{n},\left.\quad v_{n}\right|_{\partial \Omega}=0
$$

By the comparison lemma, it is clear that $u_{n} \leq v_{n}$ in $\Omega$. By regularity theory [Gu1], we may assume that $v_{n}$ converges to $v$ in $C_{0}^{1}(\bar{\Omega})$. Hence we can find a neighborhood $U$ of $\partial \Omega$ in $\Omega$ such that $u_{n} \leq v_{n} \leq \epsilon<1$.

In the following, we will show that for any point $x_{0} \in \Omega-U$, we can find a small open ball $B_{R}:=B_{x_{0}}:=B_{x_{0}}(R)$ centered at $x_{0}$ with radius $R$ such that $u_{n} \leq 1-\epsilon / 2$ on $B_{R}$ for all large $n$. Covering $\Omega-U$ by finite many such balls then gives us the conclusion that $u_{n} \leq 1-\epsilon / 2$ on $\Omega-U$. Hence we get a contradiction.

Since we are in the case $N=2$, we can choose $R>0$ such that

$$
\int_{\partial B_{x_{0}}(R)}\left|D u_{n}\right|^{p} d x \leq C
$$

and

$$
\int_{\partial B_{x_{0}}(R)}|D u|^{p} d x \leq C .
$$

Now, we let $\bar{u}_{n}$ be the harmonic function extension of $\left.u_{n}\right|_{\partial B_{R}}$ and let $\bar{u}_{\infty}$ be the harmonic function extension of $\left.u_{\infty}\right|_{\partial B_{R}}$. By the maximum principle we know that $\left|\bar{u}_{n}\right|_{L^{\infty}\left(B_{R}\right)} \leq 1\left|\bar{u}_{\infty}\right|_{L^{\infty}\left(B_{R}\right)} \leq 1$. We also have the following standard estimates: There is a uniform constant $g_{0}$ such that

$$
\left|\bar{u}_{n}(x)-\bar{u}_{n}\left(x^{\prime}\right)\right| \leq g_{0}\left|x-x^{\prime}\right|^{(p-1) / p}
$$

and

$$
\left|\bar{u}_{\infty}(x)-\bar{u}_{\infty}\left(x^{\prime}\right)\right|_{22} \leq g_{0}\left|x-x^{\prime}\right|^{(p-1) / p}
$$


for all $x, x^{\prime} \in B_{R}$. Since, for any fixed small $R_{0}>0$,

$$
\int_{B_{R_{0}}}\left|u_{n}-u_{\infty}\right| d x \leq\left|u_{n}-u_{\infty}\right|_{L^{1}} \rightarrow 0
$$

as $n \rightarrow \infty$, we have

$$
\int_{\partial B_{R}}\left|u_{n}-u_{\infty}\right| d x \rightarrow 0,
$$

for almost every $R \in\left(0, R_{0}\right)$. Hence, using Poisson's expressions for the harmonic functions $\bar{u}_{n}$ and $\bar{u}_{\infty}$, we have

$$
\left|\bar{u}_{n}-\bar{u}_{\infty}\right|(x) \rightarrow 0
$$

uniformly in $B_{R}$.

Let $w_{n}$ be the unique solution to the problem on the ball $B_{R}$ :

$$
-\Delta_{p} w_{n}=\lambda,\left.\quad w_{n}\right|_{\partial B_{R}}=u_{n}
$$

and Let $w_{\infty}$ be the unique solution to the problem on the ball $B_{R}$ :

$$
-\Delta_{p} w_{\infty}=\lambda,\left.\quad w_{\infty}\right|_{\partial B_{R}}=u_{\infty}
$$

The uniqueness of the solutions are obtained by the comparison lemma (see [Va].)The existence of $w_{n}$ can be obtained by minimize the following functional

$$
D_{p}(u)=\frac{1}{p} \int_{B_{R}}|D u|^{p} d x-\lambda \int_{B_{R}} u d x
$$

over the space $\overline{u_{n}}+W_{0}^{1, p}\left(B_{R}\right)$. In fact, the functional $D_{p}(\cdot)$ is easily seen bounded below by using the Sobolev inequality and $D_{p}(\cdot) \in C^{1}\left(W^{1, p}\left(B_{R}\right)\right)$ is weakly lower semi-continuous in $W^{1, p}\left(B_{R}\right)$. So there is a minimizer of $D_{p}(\cdot)$ on the space $\overline{u_{n}}+$ $W_{0}^{1, p}\left(B_{R}\right)$, and the minimizer is our $w_{n}$. In the same way, we find the existence and uniqueness of $w_{\infty}$. By the regularity theory $[\mathrm{T}]$ we know that they $C^{1}$ up to boundary of $B_{R}$. Note that $\lambda \geq \lambda\left|u_{n}\right|^{p-2} u_{n}$ on $B_{R}$. By the comparison lemma we get that $w_{n} \geq u_{n}$ and $w_{\infty} \geq u_{\infty}$ on $B_{R}$. Solve the problem

$$
-\Delta_{p} w=\lambda,\left.\quad w\right|_{\partial B_{R}}=1-\epsilon / 4
$$

We find that $w(x)=\lambda^{1 /(p-1)}\left(R^{p /(p-1)}-|x|^{p /(p-1)}\right)+1-\epsilon / 4$, which is less than $1-\epsilon / 8$ provided $R>0$ is small enough. Recall that our assumption on $u_{\infty}$ is $0 \leq u_{\infty}(x) \leq 1-\epsilon$ on $\Omega$. So by the comparison lemma again we find that $w \geq w_{\infty}$ on $B_{R}$. We now that $u_{n} \leq w_{n}=w_{n}-w_{\infty}+w_{\infty} \leq w_{n}-w_{\infty}+w$ on $B_{R}$. We only need to show that $w_{n}-w_{\infty}$ is small in $L^{\infty}\left(B_{R}\right)$. 
Set $\sigma=(p-1) / p$. Using a priori estimates of P. Tolksdorff ( see Proposition 6 in [To], page 144), we have a uniform constant $C_{1}>0$ such that

$$
\left|w_{n}-\bar{u}_{n}\right|(x) \leq C_{1} R^{\sigma}, \text { in } B_{R}
$$

and

$$
\left|w_{\infty}-\bar{u}_{\infty}\right|(x) \leq C_{1} R^{\sigma}, \text { in } B_{R}
$$

We remark that all the assumptions in Proposition 6 in $[\mathrm{T}]$ are satisfied except that P.Tolksdorf used the cube $Q$ not our ball. But his argument clearly works for our case. We just replace his $g$ by our harmonic functions $\bar{u}_{n}$ and $\bar{u}_{\infty}$.

By using the triangle inequality we have

$$
\begin{aligned}
\left|w_{n}-w_{\infty}\right|(x) & \leq\left|w_{n}-\bar{u}_{n}\right|(x)+\left|w_{\infty}-\bar{u}_{\infty}\right|(x)+\left|\bar{u}_{n}-\bar{u}_{\infty}\right|(x) \\
& \leq 2 C_{1} R^{\sigma}+\left|\bar{u}_{n}-\bar{u}_{\infty}\right|(x), \text { in } B_{R}
\end{aligned}
$$

which can be made arbitrarily small, saying less than $\epsilon / 100$ by choosing $R>0$ sufficiently small, for all large $n$. We remark that all the assumptions in Proposition 6 in [T] are satisfied except that P.Tolksdorf used the cube $Q$ not our ball. But his argument clearly works for our case.

Hence we have $\left|u_{n}\right|(x) \leq 1-\epsilon / 2$ on $\Omega$.

This completes the proof.

\section{REFERENCES}

[AT] S. Alama and G. Tarantello, On the solvability of a semilinear elliptic equation via an associated eigenvalue problem, Math. Z., 221 (1996), 467-493.

[AM] P. Aviles and R. McOwen, Conformal deformation to constant negative scalar curvature on noncompact Riemannian manifolds, J. Diff. Geom., 27 (1988), 225-239.

[CDG] A. Cañada, P. Drábek and J.L. Gamez, Existence of positive solutions for some problems with nonlinear diffusion, Tran. Amer. Math. Soc., 349 (1997), 4231-4249.

[Da] E.N. Dancer, Some remarks on classical problems and fine properties of Sobolev spaces, Diff. Integral Eqns., 9 (1996), 437-446.

[DD] E.N. Dancer and Y. Du, On a free boundary problem arising from population biology, Indiana Univ. Math. J., 52 (2003), 51-68.

[DDM] E.N. Dancer, Y. Du and L. Ma, Asymptotic behavior of positive solutions of some elliptic problems, Pacific J. Math., 210 (2003), 215-228.

[DG1] Y. Du and Z.M. Guo, Blow-up solutions and their applications in quasilinear elliptic equations, J. Analyse Math., 89 (2003), 277-302.

[DG2] Y. Du and Z.M. Guo, Liouville type results and eventual flatness of positive solutions for p-Laplacian equations, Adv. Differential Equations, 7 (2002), 1479-1512.

[dP] M.A. del Pino, Positive solutions of a semilinear elliptic equation on a compact manifold, Nonlinear Anal., 22 (1994), 1423-1430.

[FKLM] J.M. Fraile, P. Koch Medina, J. Lopez-Gomez and S. Merino, Elliptic eigenvalue problems and unbounded continua of positive solutions of a semilinear elliptic equation, J. Differential Equations, 127 (1996), 295-319. 
[Gu1] Z.M. Guo, Some existence and multiplicity results for a class of quasilinear elliptic eigenvalue problems, Nonlinear Anal., 18 (1992), 957-971.

[Gu2] Z.M. Guo, Uniqueness and flat core of positive solutions for quasilinear eigenvalue problems in general smooth domains, Math. Nachr., 143 (2002), 43-74.

[Gu3] Z.M. Guo, Solutions with boundary-layers and spike-layers to singularly perturbed quasilinear Dirichlet problems, Math. Nachr., (to appear).

[GW1] Z.M. Guo and J.R.L. Webb, Uniqueness of positive solutions for quasilinear elliptic equations when a parameter is large, Proc. R. Soc. Edinb., A 124 (1994), 189-198.

[GW2] Z.M. Guo and J.R.L. Webb, Large and small solutions of a class of quasilinear elliptic eigenvalue problems, J. Differential Equations, 180 (2002), 1-50.

[GZ] Z.M. Guo and H. Zhang, On the global structure of the set of positive solutions for some quasilinear elliptic boundary value problems, Nonlinear Anal., 46 (2001), 1021-1037.

[GZh] Z.M. Guo and Z.T. Zhang, $W^{1, p}$ versus $C^{1}$ local minimizers and multiplicity results for quasilinear elliptic equations, J. Math. Anal. Appl., 286 (2003), 32-50.

[He] L. Hedberg, Spectral synthesis and stability in Sobolev spaces, in Lecture Notes in Math., Vol 779, Springer-Verlag, 1980, 73-103.

[KW] J.L. Kazdan and F.W. Warner, Scalar curvature and conformal deformation of Riemannian structure, J. Diff. Geom., 10 (1975), 113-134.

[Ma] L. Ma, Conformal deformations on a noncompact Riemannian manifold, Math. Ann., 295 (1993), 75-80.

[Ou] T. Ouyang, On the positive solutions of semilinear equation $\Delta u+\lambda u-h u^{p}=0$ on the compact manifolds, Tran. Amer. Math. Soc., 331 (1992), 503-527.

[To] P. Tolksdorf, Regularity for a more general class of quasilinear elliptic equations, J. Differential Equations, 51 (1984), 126-150.

[Va] J.L. Vazquez, A strong maximum principle for some quasilinear elliptic equations, Appl. Math. Optim. 12 (1984), 191-202.

Department of Mathematics, Dong Hua University, Shanghai, 200051, P.R. China E-mail address: guozm@public.xxptt.ha.cn

Department of Mathematical Sciences, Tsinghua University, Beijing 100084, P.R. CHINA

E-mail address: Ima@math.tsinghua.edu.cn 